\title{
Temporal statistics of the beam-wander contribution to scintillation in ground-to-satellite optical links: an analytical approach
}

\author{
Alejandro Rodriguez-Gomez, Federico Dios, Juan Antonio Rubio, and Adolfo Comeron
}

\begin{abstract}
The beam-wander contribution to the scintillation in a ground-to-satellite free-space optical link is one of major importance. An analytical model, based on the duality between beam wander and angle-of-arrival fluctuations, is proposed for the temporal statistics. The expression of the probability density function of the log-amplitude fluctuations is first obtained. Then, the expressions of the spatial and temporal autocovariances are also obtained. We present plots of the beam-wander contribution to the logamplitude variance, as a function of the transmitter aperture size and the turbulence accumulated in the propagation path. We also present the angular fluctuation and log-amplitude scintillation spectrum plots for some selected cases. (C) 2005 Optical Society of America
\end{abstract}

OCIS codes: $\quad 010.1330,010.3310$.

\section{Introduction}

The atmospheric refractive turbulence is the main responsible for the limitations that arise in free-space optical communications, especially in links between ground terminals and satellites. In uplinks (between a ground-terminal transmitter and a spaceborne terminal) the effect of beam wander must be carefully considered. The authors have previously reported the results of simulations in which the importance of beam wander was stated. ${ }^{1}$

Beam wander is commonly attributed to the presence of large eddies in the turbulence structure, ${ }^{2-4}$ which cause some degree of refraction in the entire optical beam that crosses them, deviating from its original path. This deviation can produce a noticeable loss at the receiver aperture if it amounts to a significant portion of the beam width at that point.

Most of the previous literature ${ }^{4-10}$ focuses on the deduction of the variance of the angle fluctuations, without obtaining temporal statistics.

The authors are with the Department of Signal Theory and Communications, Universitat Politècnica de Catalunya, Campus Nord UPC, C/ Jordi Girona 1-3, Barcelona 08034, Spain. A. Rodriguez-Gomez can be reached by e-mail at alejandro@tsc. upc.edu.

Received 18 November 2004; revised manuscript received 28 February 2005; accepted 2 March 2005.

0003-6935/05/214574-08\$15.00/0

(C) 2005 Optical Society of America
We have made use of the Gaussian properties of the planar components of the deviation angle, and the duality between the angle-of-arrival fluctuation effect (in satellite-to-ground links) and the beam wander, as it had been proposed by Sasiela ${ }^{5}$ and Tyson. ${ }^{6}$ Thus, we can calculate spatial autocovariances and, by using Taylor's hypothesis, ${ }^{7,9}$ temporal autocovariances and spectra.

\section{Theory}

According to some authors, $, 5,6$ the beam-wander statistics can be dealt with as the dual of angle-of-arrival fluctuations in a receiver, substituting the diameter of the collecting aperture by the beam diameter at the transmitter location. This can be used to obtain the complete statistics, including the temporal ones.

\section{A. Probability Density Function}

We will call $\alpha$ the instantaneous beam deviation from the nominal pointing direction produced by the atmospheric refractive turbulence. This total angular deviation of the beam can be written as $\alpha$ $=\sqrt{\alpha_{x}^{2}+\alpha_{y}^{2}}$, where $\alpha_{x}$ and $\alpha_{y}$ are the angular deviations along two mutually perpendicular axes. If $\alpha_{x}$ and $\alpha_{y}$ are independent Gaussian random variables with zero mean, to simplify the notation, we will call $\sigma_{\alpha}{ }^{2}$ the variances of both $\alpha_{x}$ and $\alpha_{y}{ }^{7}$; thus, $\alpha$ is a Rayleigh-distributed random variable, with probability density function given by 


$$
f_{\alpha}(\alpha)=\frac{\alpha}{\sigma_{\alpha}^{2}} e^{-\frac{\alpha^{2}}{2 \sigma_{\alpha}^{2}}}
$$

Drawing upon the duality between angle-of-arrival fluctuations for a ground receiver and the beamwander fluctuations for an uplink, $\sigma_{\alpha}{ }^{2}$ can be estimated as $^{4,7-10}$

$$
\sigma_{\alpha}{ }^{2} \approx \frac{6.13}{k^{2} W_{0}^{1 / 3} r_{0}^{5 / 3}}
$$

where the receiver aperture diameter has been replaced by $\sqrt{2} W_{0}, W_{0}$ being the beam-waist radius of the assumed collimated Gaussian beam at $1 / e$ of its on-axis intensity. $k$ is the wavenumber, and $r_{0}$ is the Fried's coherence diameter, which can be calculated as $^{7}$

$$
r_{0}=\left[0.42 k^{2} \int_{0}^{L} C_{n}{ }^{2}(z) \mathrm{d} z\right]^{-3 / 5},
$$

where $C_{n}{ }^{2}(z)$ is the structure constant of the fluctuations of the index of refraction, as a function of the path $z$ and $L$ is the overall path length.

We will assume that, as far as the turbulenceinduced mispoint effect is concerned, the beam reaching the satellite can be considered as a Gaussian one with an angular intensity profile given by

$$
I(\theta)=I_{M} e^{-\frac{\theta^{2}}{\Delta^{2} \theta}},
$$

where $I_{M}$ is the maximum (on-axis) intensity and $\Delta \theta$ is the rms angular radius at $1 / e$ of the maximum intensity, taking into account the short-term beamspread effects. $\Delta \theta$ can be calculated as

$$
\Delta \theta \approx \frac{W(L)}{L}
$$

where $W(L)$ is the rms radius at $1 / e$ of the maximum intensity of the transmitted Gaussian beam at a distance $L$ and can be calculated $\mathrm{as}^{4}$ :

$$
W(L)=\left[W_{0}^{2}+\left(\frac{L \lambda}{\pi W_{0}}\right)^{2}+2\left(\frac{L \lambda}{\pi \rho_{s t}}\right)^{2}\right]^{1 / 2} .
$$

The parameter $\rho_{s t}$ corresponds to the short-term coherence radius, and it can be calculated ${ }^{11}$ as

$$
\rho_{s t}=\rho_{c}\left[1+0.33\left(\frac{\rho_{c}}{W_{0}}\right)^{1 / 3}\right] .
$$

The parameter $\rho_{c}$ corresponds to the long-term coherence radius for a collimated beam, and it can be calculated $^{12}$ as

$$
\rho_{c}=\frac{r_{0}}{2}\left[\frac{1+\frac{\theta_{0}^{2}}{2}\left(1+\frac{8 W_{0}^{2}}{3 r_{0}^{2}}\right)}{1+\frac{\theta_{0}^{2}}{6}\left(1+\frac{2 W_{0}^{2}}{r_{0}^{2}}\right)}\right]^{1 / 2},
$$

where $\theta_{0}$ is the diffraction-limited angular radius of the transmitted beam at $1 / e$ of the maximum intensity, $\theta_{0}=\lambda / \pi W_{0}$.

The intensity reaching the satellite receiver as a function of $\alpha$, the instantaneous mispointing, is

$$
I=I_{M} e^{-\frac{\alpha^{2}}{\Delta^{2} \theta}}
$$

The log-amplitude fluctuation, with respect to the maximum level, due to the turbulence-induced beam wander, is related to the instantaneous mispointing through

$$
\chi_{W}=\frac{1}{2} \ln \frac{I}{I_{M}}=-\frac{1}{2} \frac{\alpha^{2}}{\Delta^{2} \theta}=K \alpha^{2} .
$$

With the relationship $\chi_{W}=f(\alpha)$ given by Eq. (10), the probability density function of $\chi_{W}, f_{\chi_{W}}\left(\chi_{W}\right)$, can then be derived from the probability density function of $\alpha$, $f_{\alpha}(\alpha)$, using 13

$$
f_{\chi_{W}}\left(\chi_{W}\right)=\frac{f_{\alpha}\left[f^{-1}\left(\chi_{W}\right)\right]}{\left|f^{\prime}\left[f^{-1}\left(\chi_{W}\right)\right]\right|}
$$

Employing Eqs. (1) and (10) in Eq. (11), the probability density function of $\chi_{W}$ is readily found:

$$
f_{\chi_{W}}\left(\chi_{W}\right)=\left(\frac{\Delta \theta}{\sigma_{\alpha}}\right)^{2} e\left(\frac{\Delta \theta}{\sigma_{\alpha}}\right)_{\chi_{W}}^{2}, \text { with } \chi_{W} \leq 0
$$

Expression (12) is an exponential probability law with mean $-\left(\sigma_{\alpha} / \Delta \theta\right)^{2}$ and variance $\left(\sigma_{\alpha} / \Delta \theta\right)^{4}$ (see, for example, Ref. 14).

\section{B. Temporal Statistics}

To calculate the temporal autocorrelation of the logamplitude fluctuations, $C_{\chi_{W}}(\tau)$, we will use the Taylor's hypothesis, relating temporal covariance functions to spatial ones. The previous step will then be to calculate $C_{\chi_{W}}(\rho)$ at the plane of the transmitter,

$$
\begin{aligned}
C_{\chi_{W}}(\rho) & =\left\langle\left[\chi_{W}\left(\rho^{\prime}+\rho\right)-\mu_{\chi_{W}}\right]\left[\chi_{W}\left(\rho^{\prime}\right)-\mu_{\chi_{W}}\right]\right\rangle \\
& =\left\langle\chi_{W}\left(\rho^{\prime}+\rho\right) \chi_{W}\left(\rho^{\prime}\right)\right\rangle-\mu_{\chi_{W}}{ }^{2},
\end{aligned}
$$

where spatial homogeneity has been assumed. $\mu_{\chi_{W}}$ is the mean value of the log-amplitude of the fluctuation due to beam wander.

Let us concentrate on the spatial correlation function for the log-amplitude fluctuation associated with beam wander: 


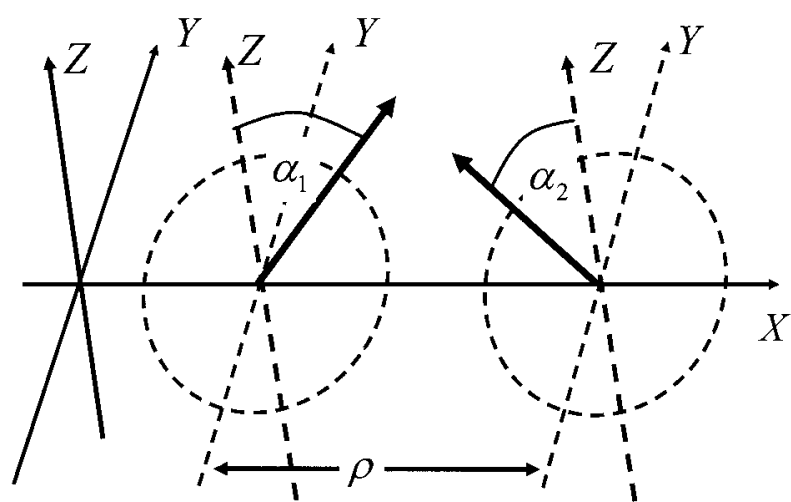

Fig. 1. Geometrical arrangement for the computation of the joint probability density of the angular fluctuations. The plane $z=0$ corresponds to the location of the transmitting aperture. We use the classical approach of considering two apertures, separated by a distance $\rho$ to calculate the spatial autocorrelation.

$$
\begin{aligned}
R_{\chi_{W}}(\rho) & =\left\langle\chi_{W}\left(\rho^{\prime}+\rho\right) \chi_{W}\left(\rho^{\prime}\right)\right\rangle \\
& =\left\langle f\left[\alpha\left(\rho^{\prime}+\rho\right)\right] f\left[\alpha\left(\rho^{\prime}\right)\right]\right\rangle,
\end{aligned}
$$

where $\chi_{W}=f(\alpha)$ given by Eq. (10) is assumed.

By definition we have

$$
\begin{aligned}
& \left\langle f\left[\alpha\left(\rho^{\prime}+\rho\right)\right] f\left[\alpha\left(\rho^{\prime}\right)\right]\right\rangle= \\
& \int_{-\infty}^{\infty} \int_{-\infty}^{\infty} f\left(\alpha_{1}\right) f\left(\alpha_{2}\right) f_{\alpha \alpha}\left(\alpha_{1}, \alpha_{2} ; \rho\right) d \alpha_{1} d \alpha_{2},
\end{aligned}
$$

where $f_{\alpha \alpha}\left(\alpha_{1}, \alpha_{2} ; \rho\right)$ is the joint probability density function for the angular deviation of beams with axes separated a distance $\rho$ at their output.

Figure 1 shows the arrangement made for the computation of the joint statistics. The transmitting aperture is located at $z=0$ plane, and a second transmitting aperture (located at a distance $\rho$ ) is considered. To determine $f_{\alpha \alpha}\left(\alpha_{1}, \alpha_{2} ; \rho\right)$ we start considering the joint probability density function for the angular deviations along perpendicular directions $X$ and $Y$ of beams with axes separated a distance $\rho$. Let us call these deviations $\alpha_{1 x}, \alpha_{1 y}$, and $\alpha_{2 x}, \alpha_{2 y}$, respectively, where $f_{\alpha \alpha}\left(\alpha_{1}, \alpha_{2} ; \rho\right)$ is the joint probability density function for the angular deviation of beams with axes separated a distance $\rho$ at their output. We obviously have

$$
\alpha_{i}=\sqrt{{\alpha_{i x}}^{2}+\alpha_{i y}^{2}} \text {, with } i=1,2 \text {. }
$$

This situation can be considered as the dual of two apertures receiving wavefronts affected by turbulence. The angles along the perpendicular directions can be considered as joint normal zero-mean random variables. Calling $\alpha_{1 x}$ and $\alpha_{1 y}$ the angular deviation along the directions $X$ and $Y$, respectively, for the beam $i(i=1,2)$, the joint probability density for the four angles under consideration can be written ${ }^{13}$

$$
\begin{aligned}
f_{\alpha_{1 x} \alpha_{1 y} \alpha_{2 x} \alpha_{2 y}}\left(\alpha_{1 x} \alpha_{1 y} \alpha_{2 x} \alpha_{2 y} ; \rho\right)= & \frac{1}{(2 \pi)^{2}|\mathbf{C}|^{1 / 2}} \\
& \times \exp \left(-\frac{\boldsymbol{\alpha}^{T} \mathbf{C}^{-1} \boldsymbol{\alpha}}{2}\right),
\end{aligned}
$$

with $\boldsymbol{\alpha}^{T}$ the vector $\boldsymbol{\alpha}^{T}=\left[\alpha_{1 x} \alpha_{1 y} \alpha_{2 x} \alpha_{2 y}\right]$ and $\mathbf{C}$ the covariance matrix with elements

$$
c_{i p j q}(\rho)=\left\langle\alpha_{i p} \alpha_{j q}\right\rangle,
$$

with $i, j-1,2$ and $p, q=x, y$. The isotropy of the turbulence entails $c_{i p j q}(\rho)=0$ for $p \neq q$, and $c_{1 x 2 x}(\rho)$ $=c_{1 u 2 u}(\rho)$. Drawing on the duality between angle of arrival and beam deviation, and working out the expression for the image centroid position in a receiving system found in Ref. 7, we obtain

$$
\begin{aligned}
c_{1 u 2 u}(\rho)= & \frac{16 \pi^{2}}{W_{0}^{2}} \times 0.033 \int_{0}^{L} C_{n}^{2}(z) \int_{0}^{\infty} K^{-8 / 3} \\
& \times J_{1}^{2}\left(\frac{W_{0}}{\sqrt{2}} K\right) J_{0}(\rho K) \mathrm{d} K \mathrm{~d} z,
\end{aligned}
$$

where $W_{0}$ is the beamwaist radius of the assumed Gaussian beam at $1 / e$ of its on-axis intensity. Obviously $c_{1 u 2 u}(0)=\sigma_{\alpha}^{2}$, the variance of the angular fluctuations of the beam axis.

The general expression (17) then reduces to

$$
\begin{aligned}
& f_{\alpha_{1 x} \alpha_{1 y} \alpha_{2 x} \alpha_{2 y}}\left(\alpha_{1 x}, \alpha_{1 y}, \alpha_{2 x}, \alpha_{2 y} ; \rho\right)= \\
& \frac{1}{(2 \pi)^{2}\left[\sigma_{\alpha}{ }^{4}-c_{1 u 2 u}{ }^{2}(\rho)\right]} e^{-\frac{p\left(\alpha_{1 x} x^{2}+\alpha_{1 y}{ }^{2}+\alpha_{2 x} x^{2}+\alpha_{2 y}{ }^{2}\right)+2 q\left(\alpha_{1 x} \alpha_{2 x}+\alpha_{1 y} \alpha_{2 y}\right)}{2}},
\end{aligned}
$$

with

$$
\begin{aligned}
& p=\frac{\sigma_{\alpha}{ }^{2}}{\sigma_{\alpha}{ }^{4}-c_{1 u 2 u}{ }^{2}(\rho)}, \\
& q=-\frac{c_{1 u 2 u}(\rho)}{\sigma_{\alpha}{ }^{4}-c_{1 u 2 u}{ }^{2}(\rho)} .
\end{aligned}
$$

We can derive the joint probability density function of $\alpha_{1}$ and $\alpha_{2}$ by noting that

$$
\begin{gathered}
\alpha_{1 x}{ }^{2}+\alpha_{1 y}{ }^{2}+\alpha_{2 x}{ }^{2}+\alpha_{2 y}{ }^{2}=\alpha_{1}{ }^{2}+\alpha_{2}{ }^{2}, \\
\alpha_{1 x} \alpha_{2 x}+\alpha_{1 y} \alpha_{2 y}=\alpha_{1} \alpha_{2} \cos \left(\phi-\phi^{\prime}\right) .
\end{gathered}
$$

We take into account that the joint probability density function of $\alpha_{1}$ and $\alpha_{2}$ is defined by 


$$
\begin{aligned}
& f_{\alpha \alpha}\left(\alpha_{1}, \alpha_{2} ; \rho\right) \mathrm{d} \alpha_{1} \mathrm{~d} \alpha_{2}= \\
& \quad p\left\{\alpha_{1}<\alpha_{1}{ }^{\prime}<\alpha_{1}+\mathrm{d} \alpha_{1}, \alpha_{2}<\alpha_{2}{ }^{\prime}<\alpha_{2}+\mathrm{d} \alpha_{2}\right\},
\end{aligned}
$$

and substituting Eqs. (23) and (24) into Eq. (20), we find

$$
\begin{aligned}
f_{\alpha \alpha}\left(\alpha_{1}, \alpha_{2} ; \rho\right) \mathrm{d} \alpha_{1} \mathrm{~d} \alpha_{2} \\
=\frac{1}{(2 \pi)^{2}\left[\sigma_{\alpha}{ }^{4}-c_{1 u 2 u}{ }^{2}(\rho)\right]} \alpha_{1} \alpha_{2} e^{-\frac{p\left(\alpha_{1}{ }^{2}+\alpha_{2} 2\right)}{2}} \\
\quad \times \int_{0}^{2 \pi} \int_{0}^{2 \pi} e^{-q \alpha_{1} \alpha_{2} \cos \left(\phi-\phi^{\prime}\right)} \mathrm{d} \phi \mathrm{d} \phi^{\prime} \mathrm{d} \alpha_{1} \mathrm{~d} \alpha_{2} .
\end{aligned}
$$

But

$$
\int_{0}^{2 \pi} e^{-q \alpha_{1} \alpha_{2} \cos \left(\phi-\phi^{\prime}\right)} \mathrm{d} \phi=2 \pi I_{0}\left(-q \alpha_{1} \alpha_{2}\right),
$$

and substituting Eq. (27) into Eq. (26), we obtain

$$
\begin{aligned}
f_{\alpha \alpha}\left(\alpha_{1}, \alpha_{2} ; \rho\right)= & \frac{1}{\sigma_{\alpha}{ }^{4}-c_{1 u 2 u}{ }^{2}(\rho)} \alpha_{1} \alpha_{2} \\
& \times \exp \left\{-\frac{\sigma_{\alpha}{ }^{2}\left(\alpha_{1}{ }^{2}+\alpha_{2}{ }^{2}\right)}{2\left[\sigma_{\alpha}{ }^{4}-c_{1 u 2 u}{ }^{2}(\rho)\right]}\right\} \\
& \times I_{0}\left[\frac{c_{1 u 2 u}(\rho)}{\sigma_{\alpha}{ }^{4}-c_{1 u 2 u}{ }^{2}(\rho)} \alpha_{1} \alpha_{2}\right],
\end{aligned}
$$

where the expressions (21) and (22) for $p$ and $q$ have been substituted. Expression (28) corresponds to a joint Rayleigh probability density function. ${ }^{15}$

Substituting expressions (10) and (28) into (15), one finds

$$
\begin{aligned}
R_{\chi_{W}}(\rho)= & \frac{1}{4 \Delta^{4} \theta\left[\sigma_{\alpha}{ }^{4}-c_{1 u 2 u}{ }^{2}(\rho)\right]} \\
& \times \int_{0}^{\infty} \int_{0}^{\infty} \alpha_{1}{ }^{3} \alpha_{2}{ }^{3} \exp \left\{-\frac{\sigma_{\alpha}{ }^{2}\left(\alpha_{1}{ }^{2}+\alpha_{2}{ }^{2}\right)}{2\left[\sigma_{\alpha}{ }^{4}-c_{1 u 2 u}{ }^{2}(\rho)\right]}\right\} \\
& \times I_{0}\left[\frac{c_{1 u 2 u}(\rho)}{\sigma_{\alpha}{ }^{4}-c_{1 u 2 u}{ }^{2}(\rho)} \alpha_{1} \alpha_{2}\right] \mathrm{d} \alpha_{1} \mathrm{~d} \alpha_{2}
\end{aligned}
$$

which after some rather cumbersome manipulations (that can be found in Appendix A) yields the surpris- ingly simple expression

$$
R_{\chi W}(\rho)=\frac{1}{\Delta^{4} \theta}\left[\sigma_{\alpha}^{4}+c_{1 u 2 u}{ }^{2}(\rho)\right] .
$$

Then, using Eq. (13) and taking into account that $\mu_{\chi_{W}}=-\sigma_{\alpha}{ }^{2} / \Delta^{2} \theta$, the following expression is found for the spatial covariance function of the power logamplitude fluctuations due to the beam wander:

$$
C_{\chi_{W}}(\rho)=\frac{c_{1 u 2 u}{ }^{2}(\rho)}{\Delta^{4} \theta} .
$$

Using the Taylor's hypothesis, the temporal covariance of the log-amplitude fluctuations due to beam wander is found by replacing $\rho \rightarrow \nu \tau$ in Eq. (31), with $v$ the transverse component of wind velocity, to obtain

$$
C_{\chi_{W}}(\tau)=\frac{c_{1 u 2 u}{ }^{2}(\tau)}{\Delta^{4} \theta}
$$

where an abuse of language has been tolerated when using the same symbols to denote spatial and temporal covariances.

From Eq. (32), the temporal spectrum of logamplitude fluctuations due to the turbulence-induced beam wander can be computed through the Fourier transform.

\section{Numerical Example}

We have performed some numerical computations to predict the beam-wander values of an optical link established between a transmitter located in a ground station and a geostationary satellite. A complete description of one actual implementation of such terminals can be found in Refs. 16 and 17.

The turbulence has been characterized according to the results of a measurement campaign that took place in the Izaña observatory, Tenerife Island, Spain, in May 1995.18,19 For weak turbulence conditions, the structure constant of the index of refraction fluctuations can be modeled by the following expres$\operatorname{sion}^{7}$ :

$$
C_{n}^{2}(h)= \begin{cases}C_{n 0}{ }^{2}\left(\frac{h}{h_{s}}\right)^{-2 / 3} & h \leq h_{s} \\ C_{n 0}{ }^{2} & h_{s}<h<h_{i}, \\ C_{n 0}{ }^{2} e^{\frac{h_{i}}{h_{r}}} e^{-\frac{h}{h_{r}}}+C_{n l}{ }^{2} e^{-\frac{h}{h_{l}}}+C_{n l}{ }^{2} 3 W^{2}\left(\frac{h}{h_{t}}\right)^{10} e^{-\frac{10 h}{h_{t}}} & h>h_{i}\end{cases}
$$




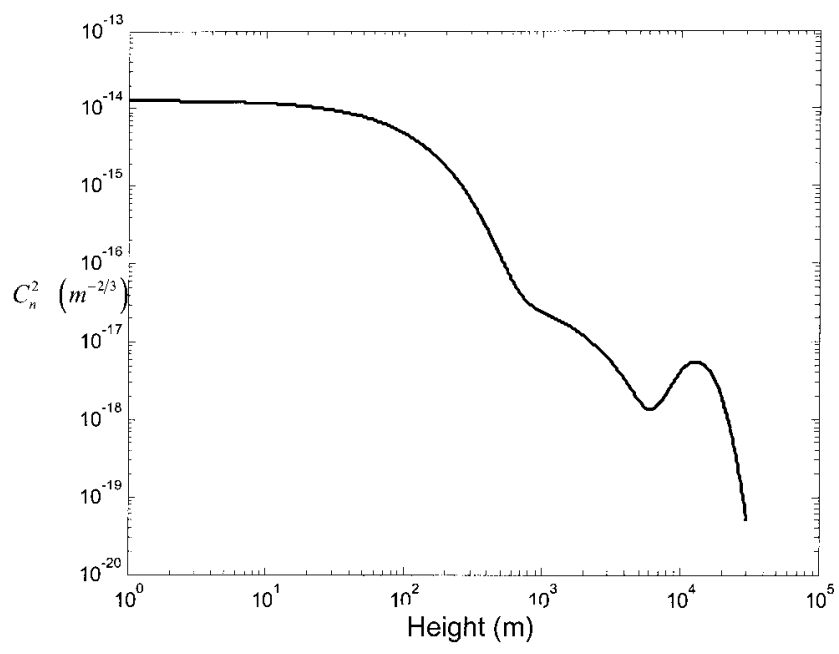

Fig. 2. Structure constant of the fluctuations of the index of refraction as a function of height over sea level, using Ref. 18.

where $h$ is the height above the terrain; $h_{s}=4 \mathrm{~m}$ is the surface layer height (typically of few meters); $C_{n 0}{ }^{2}=9.5 \times 10^{-15} \mathrm{~m}^{-2 / 3}$ is the structure constant value assumed uniform throughout the inversion layer extending between the heights $h_{s}$ and $h_{i}$ $=30 \mathrm{~m} ; C_{n l}^{2}=4.5 \times 10^{-17} \mathrm{~m}^{-2 / 3}$ is the structureconstant characteristic value for free atmosphere; $h_{l}$ $=1,500 \mathrm{~m}$ is the height characterizing the exponential fall-off of the structure constant in the freeatmosphere region; $W=30 \mathrm{~m} / \mathrm{s}$ is the root-meansquared wind velocity averaged over the 5 to $20 \mathrm{~km}$ altitude interval, and $h_{t}=13,000 \mathrm{~m}$ is the tropopause height. Figure 2 shows the dependence of $C_{n}^{2}$ with height over sea level.

We have considered several values for the elevation angle of the link path: $5^{\circ}, 15^{\circ}, 30^{\circ}, 45^{\circ}, 60^{\circ}$, and $90^{\circ}$, and transmitting aperture diameters between $1 \mathrm{~cm}$ and $1 \mu \mathrm{m}$. For the application of the Taylor hypothesis, a $5 \mathrm{~m} / \mathrm{s}$ wind speed has been considered. A $1 \mu \mathrm{m}$ wavelength was used in all the computations.

Figure 3 shows the variation of the beam angular width with the transmitter aperture diameter, for different elevation values of the link path. For small values of the diameter, the behavior is that from a Gaussian beam: the angular width falls with increasing diameter, showing that the turbulence effect is very weak. This decreasing behavior stops at a given value (that depends on the length of the path inside the atmosphere and, thus, on the elevation angle) and remains constant, revealing that the turbulence effect is limiting the effective aperture.

The combination of the results of the beam-wander angular variance (according to expression (2)) with the values shown in Fig. 3 can be used to compute the variance of the log-amplitude fluctuations of the optical intensity at the receiver plane due to the beamwander effect, as it was expressed in Subsection 2.A, after Eq. (12) or also according to expression (30), taking $\rho=0$. Figure 4 shows the dependence with the transmitter diameter, for different elevation angles. The result shows, coherently with Fig. 3, a pesimum

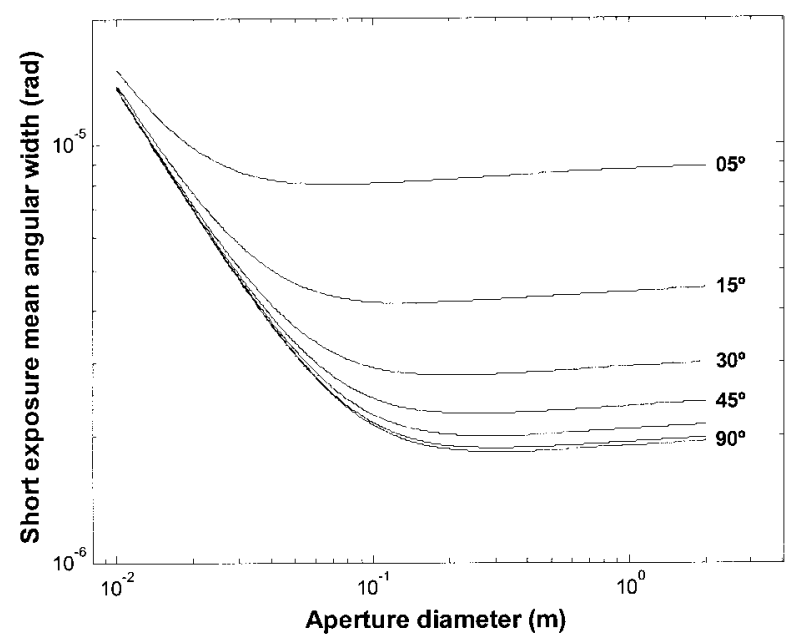

Fig. 3. Short-exposure beam-spread mean value, as a function of the transmitter aperture size, for different path elevation angles.

value for the aperture diameter, which depends on the elevation angle of the link path and is responsible for the behavior that is observed in the following figures. The presence of a pesimum effect has also been reported by Baker et al. ${ }^{20}$

Figures 5, 6, and 7 show the temporal spectra of the angular fluctuations for different aperture diameters $(1 \mathrm{~cm}, 10 \mathrm{~cm}$, and $1 \mathrm{~m})$ and considering different elevation angles $\left(5^{\circ}, 15^{\circ}, 30^{\circ}, 45^{\circ}, 60^{\circ}, 75^{\circ}\right.$, and $\left.90^{\circ}\right)$. These spectra have been obtained from the spatial covariance predicted by Eq. (19), in which the temporal dependence has been introduced according to the Taylor hypothesis for a $5 \mathrm{~m} / \mathrm{s}$ wind speed. All three figures show increasing values for decreasing elevation angles. For the higher elevation angles, the plots tend to accumulate. The quickest variations are present for smaller transmitter size, leading to wider spectra; for wider apertures, the spectra are narrower but, as the contribution to variance is smaller, ac-

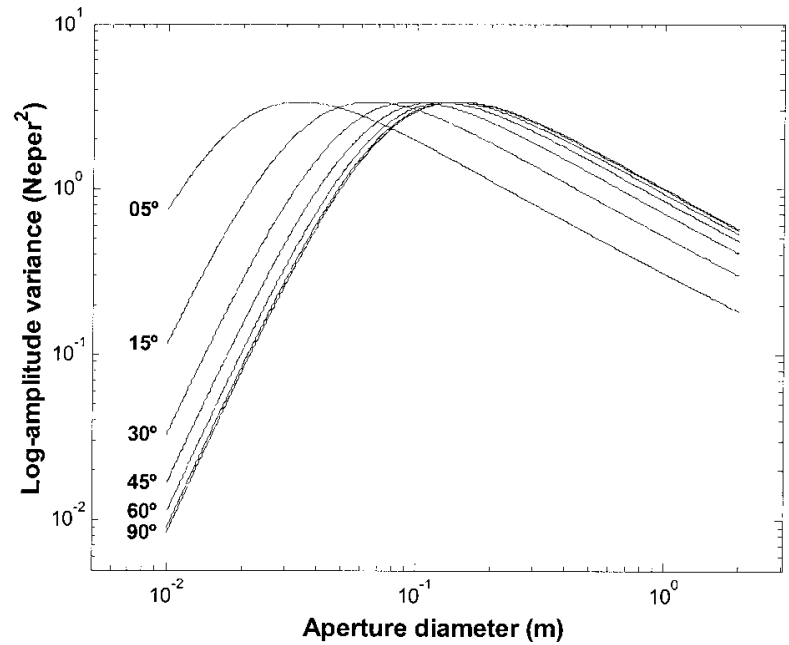

Fig. 4. Beam-wander contribution to scintillation variance at the spaceborne receiver, as a function of the transmitter aperture size, for different path elevation angles. 


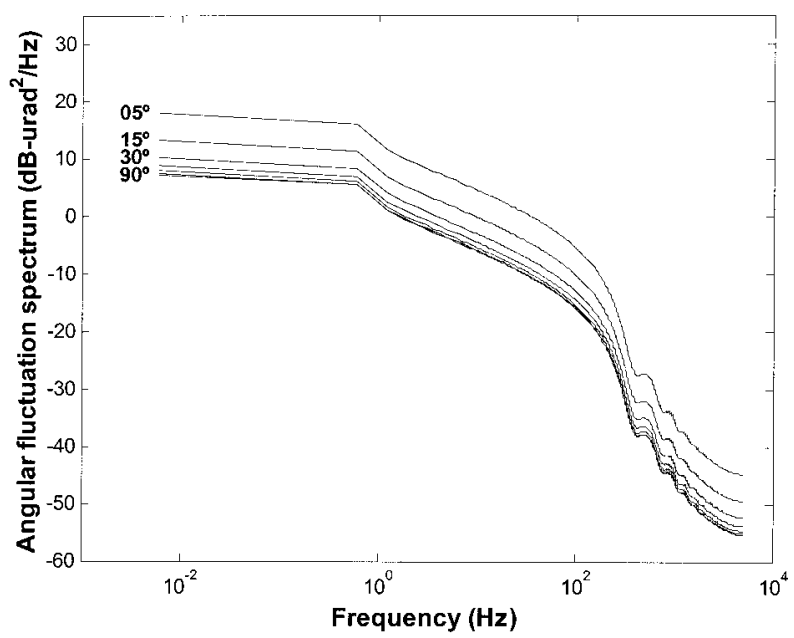

Fig. 5. Angular fluctuation spectrum for a $1 \mathrm{~cm}$ diameter transmitter aperture, $5 \mathrm{~m} / \mathrm{s}$ wind speed, and for different path elevation angles.

cordingly to Eq. (2), the spectral values do not grow significantly.

More surprising effects can be appreciated in the plots showing the spectra of the log-amplitude fluctuations at the receiver aperture. Figure 8 shows the spectra for a $1 \mathrm{~cm}$ diameter transmitting aperture; accordingly with the angular fluctuation presented in Fig. 5 and the beam spread presented in Fig. 3, the values increase with the descending elevation angle. Meanwhile, Fig. 9 show how for a $1 \mathrm{~m}$ transmitter the fluctuation is stronger (although the variation is smaller than in Fig. 8) for higher elevation angles. This effect is consistent with the inversion that was presented in Fig. 4: for big apertures, the beam spread does not reduce, so that the effect of beam wander is compensated. Figure 9 shows an intermediate situation located barely in the center of the different pesima: the fluctuation is worse for the higher angles, but all the plots are very close.

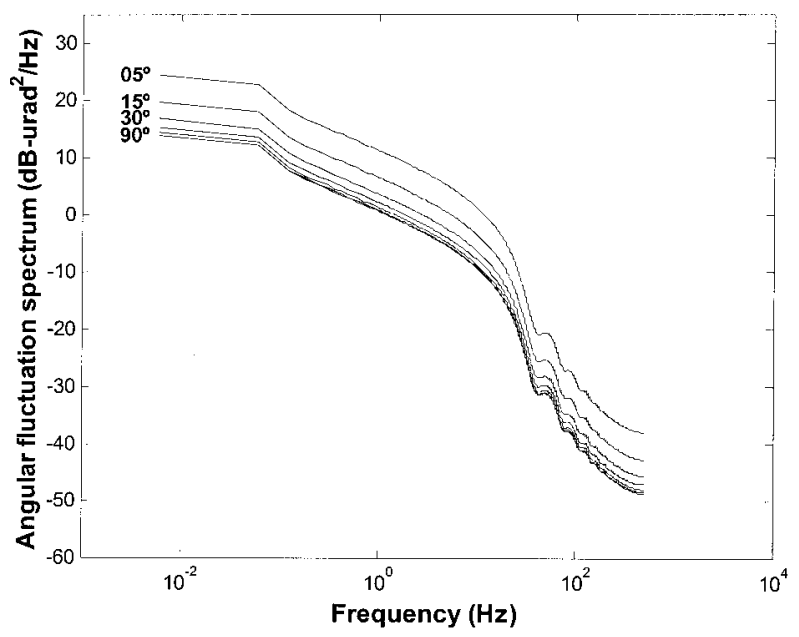

Fig. 6. Angular fluctuation spectrum for a $10 \mathrm{~cm}$ diameter transmitter aperture, $5 \mathrm{~m} / \mathrm{s}$ wind speed, and for different path elevation angles.

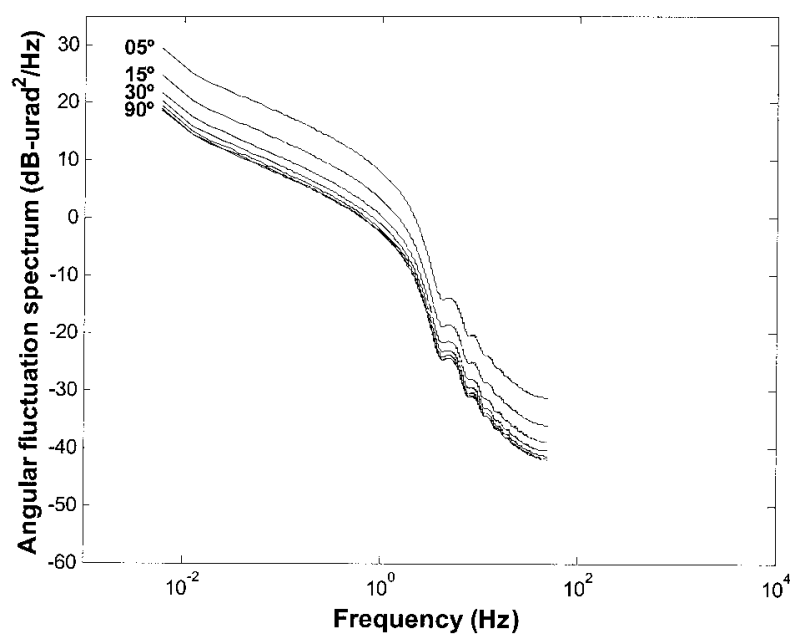

Fig. 7. Angular fluctuation spectrum for a $1 \mathrm{~m}$ diameter transmitter aperture, $5 \mathrm{~m} / \mathrm{s}$ wind speed, and for different path elevation angles.

Generally speaking, the intermediate aperture values appear to show the worst scintillation behavior. From the point of view of the beam-wander contribution, increasing the aperture size is the way to maximize the transmitter gain (although it saturates, as shown in Fig. 3) and to minimize the scintillation.

\section{Conclusions}

We have presented an analytical formalism that models the beam-wander effect in free-space optical communications when the turbulence concentrates near the transmitter, as happens in communications between the Earth's surface and a satellite. The model considers the beam movement as the dual of the fluctuations of the angle-of-arrival in a receiver with the turbulence concentrated on its side, as it had been proposed by Sasiela. ${ }^{5}$ From this starting point, we present how nontemporal and temporal statistics

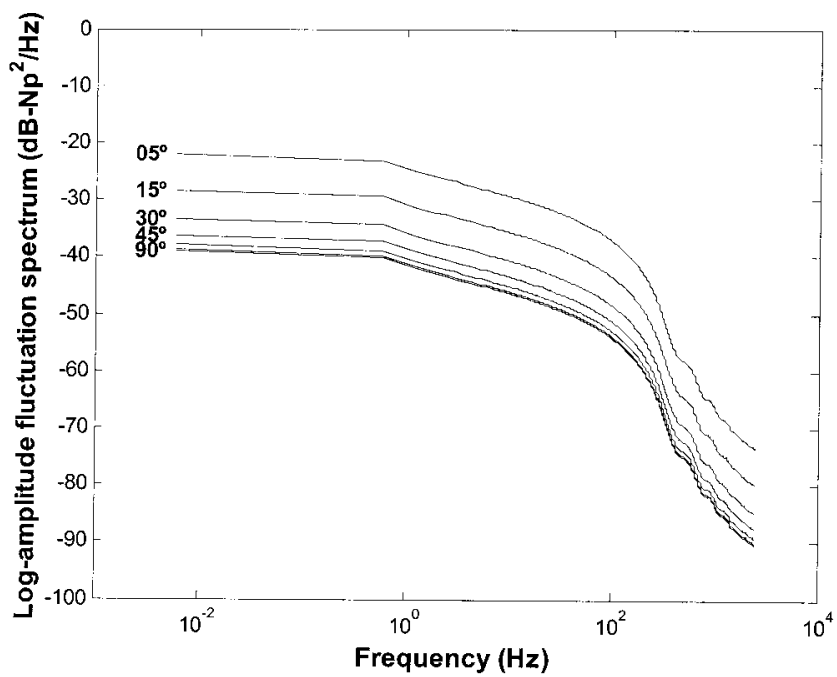

Fig. 8. Spectrum beam-wander contribution to scintillation for a $1 \mathrm{~cm}$ diameter transmitter aperture, $5 \mathrm{~m} / \mathrm{s}$ wind speed, and for different path elevation angles. 


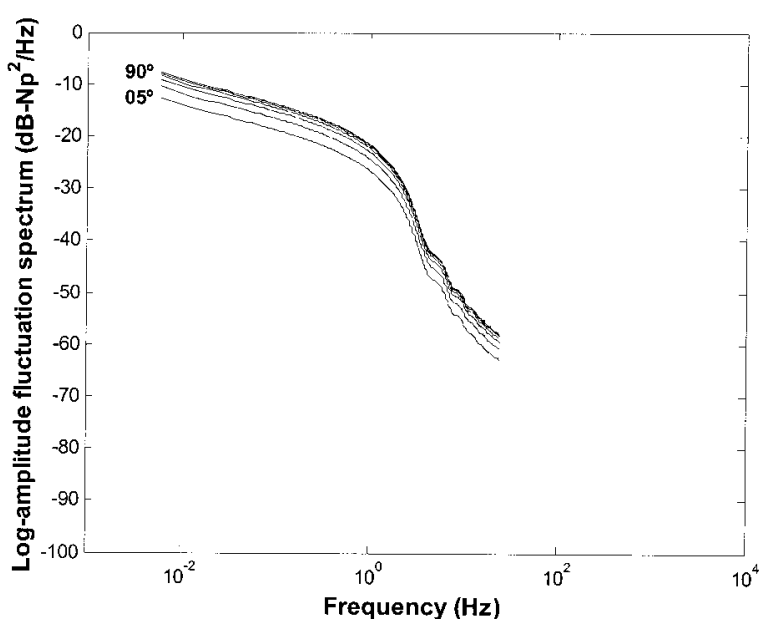

Fig. 9. Spectrum beam-wander contribution to scintillation for a $1 \mathrm{~m}$ diameter transmitter aperture, $5 \mathrm{~m} / \mathrm{s}$ wind speed, and for different path elevation angles.

can be obtained. Finally we have presented a numerical example in which the effect of the accumulation of turbulence is observed for different transmitter aperture values.

\section{Appendix A: Derivation of Expression (30)}

Let us consider the evaluation of the integral in expression (29). First, we will call

$$
s=\sigma_{\alpha}{ }^{4}-c_{12}{ }^{2}(\rho) .
$$

And then, we concentrate on the inner integral in Eq. (29):

$$
\int_{0}^{\infty} \alpha_{2}{ }^{3} \exp \left(-\frac{\sigma_{a}{ }^{2} \alpha_{2}^{2}}{2 s}\right) I_{0}\left(\frac{c_{12}}{s} \alpha_{1} \alpha_{2}\right) \mathrm{d} \alpha_{2}
$$

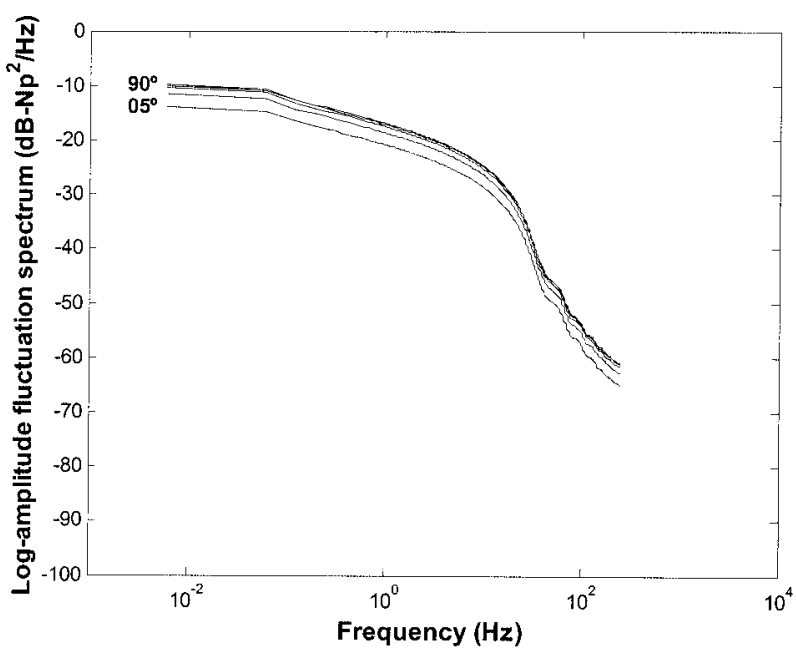

Fig. 10. Spectrum beam-wander contribution to scintillation for a $10 \mathrm{~cm}$ diameter transmitter aperture, $5 \mathrm{~m} / \mathrm{s}$ wind speed, and for different path elevation angles. where the dependence of $s$ and $c_{12}$ on $\rho$ is not explicitly shown. By substituting in integral (35),

$$
\begin{aligned}
\alpha_{2}^{2} & =x, \\
\mathrm{~d} \alpha_{2} & =\frac{1}{2} x^{-1 / 2} \mathrm{~d} x .
\end{aligned}
$$

And using the relationship between the standard and the modified Bessel functions, ${ }^{21}$ we can write

$$
\frac{1}{2} \int_{0}^{\infty} x \exp \left(-\frac{\sigma_{\alpha}^{2} x}{2 s}\right) J_{0}\left(i \frac{c_{12}}{s} \alpha_{1} x^{1 / 2}\right) \mathrm{d} x
$$

This integral can be solved using the equivalence ${ }^{21}$

$$
\begin{aligned}
& \int_{0}^{\infty} x^{n+\frac{1}{2} v} \exp (-\alpha x) J_{v}(2 \beta \sqrt{x}) \mathrm{d} x= \\
& n ! \beta^{v} \exp \left(-\frac{\beta^{2}}{\alpha}\right) \alpha^{-n-v-1} L_{n}^{v}\left(\frac{\beta^{2}}{\alpha}\right),
\end{aligned}
$$

where $L_{n}{ }^{v}(z)$ is a Laguerre polynomial.

Comparing our integral with the model, we find the following correspondences:

$$
\begin{aligned}
& n=1, \alpha=\frac{\sigma_{a}{ }^{2}}{2 s}, \\
& \beta=i \frac{c_{12} \alpha_{1}}{2 s}, \nu=0,
\end{aligned}
$$

For these values, the Laguerre polynomial is ${ }^{21}$

$$
L_{1}^{0}(z)=1-z .
$$

So the integral in expression (37) can be calculated as

$$
s^{2} \frac{2}{\sigma_{\alpha}{ }^{4}} \exp \left[\left(\frac{c_{12}}{\sigma_{\alpha}}\right)^{2} \frac{\alpha_{1}^{2}}{2 s}\right]\left[1+\left(\frac{c_{12}}{\sigma_{\alpha}}\right)^{2} \frac{\alpha_{1}^{2}}{2 s}\right]
$$

Then we can calculate the complete expression (29) as

$$
\begin{aligned}
R_{\chi_{W}}(\rho)= & \frac{2 K^{2} s}{\sigma_{\alpha}{ }^{4}}\left[\int_{0}^{\infty} \alpha_{1}^{3} \exp \left(-\frac{\alpha_{1}^{2}}{2 \sigma_{a}^{2}}\right) \mathrm{d} \alpha_{1}\right. \\
& \left.+\frac{1}{2 s}\left(\frac{c_{12}}{\sigma_{\alpha}}\right)^{2} \int_{0}^{\infty} \alpha_{1}^{5} \exp \left(-\frac{\alpha_{1}^{2}}{2 \sigma_{a}^{2}}\right) \mathrm{d} \alpha_{1}\right]
\end{aligned}
$$

Now we introduce new variable changes,

$$
\alpha_{1}^{2}=x, \mathrm{~d} \alpha_{1}=\frac{1}{2} x^{-1 / 2} \mathrm{~d} x,
$$


obtaining

$$
\begin{aligned}
R_{\chi_{W}}(\rho)= & \frac{K^{2} s}{\sigma_{\alpha}{ }^{4}}\left[\int_{0}^{\infty} x \exp \left(-\frac{x}{2 \sigma_{a}^{2}}\right) \mathrm{d} x+\frac{1}{2 s}\left(\frac{c_{12}}{\sigma_{\alpha}}\right)^{2}\right. \\
& \left.\times \int_{0}^{\infty} x^{2} \exp \left(-\frac{x}{2 \sigma_{a}^{2}}\right) \mathrm{d} x\right] .
\end{aligned}
$$

Once again we can use a tabulated integral from Ref. 21:

$$
\int_{0}^{\infty} x^{n} \exp (-\mu x) \mathrm{d} x=n ! \mu^{-n-1} .
$$

And finally, substituting the different equivalences, we obtain

$$
R_{\chi W}(\rho)=\frac{1}{\Delta^{4} \theta}\left[\sigma_{\alpha}^{4}+c_{12}{ }^{2}(\rho)\right] .
$$

This work has been funded by the European Space Agency through project 16021/02/NL/CK. We would like to thank Zoran Sodnik for his encouragement. We also would like to thank Marcos Reyes and all his team at the Instituto Astrofísico de Canarias.

\section{References}

1. F. Dios, J. A. Rubio, A. Rodriguez, and A. Comeron, "Scintillation and beam-wander analysis in an optical ground stationsatellite uplink," Appl. Opt. 43, 3866-3873 (2004).

2. L. C. Andrews and R. L. Phillips, Laser Beam Propagation through Random Media, Vol. PM53 of SPIE Press Monograph Series (SPIE, Bellingham, Wash., 1998).

3. J. H. Churnside and R. J. Lataitis, "Wander of an optical beam in the turbulent atmosphere," Appl. Opt. 29, 926-930 (1990).

4. R. L. Fante, "Electromagnetic beam propagation in turbulent media," Proc. IEEE 63, 1669-1692 (1975).

5. R. J. Sasiela, Electromagnetic Wave Propagation in Turbulence. Evaluation and Application of Mellin Transforms (Springer-Verlag, Berlin, 1994).
6. R. K. Tyson, Principles of Adaptive Optics (Academic, Boston, 1998).

7. R. E. Hufnagel, "Propagation through atmospheric turbulence," in The Infrared Handbook, W. L. Wolfe and G. J. Zissis, eds. (Office of Naval Research, Washington, D.C., 1978).

8. R. R. Beland, "Propagation through atmospheric optical turbulence," in Atmospheric Propagation of Radiation, Vol. 2 of The Infrared \& Electro-Optical Systems Handbook, F. G. Smith, ed. (SPIE, Bellingham, Wash., 1993).

9. A. Ishimaru, "The beam wave case and remote sensing," in Laser Beam Propagation in the Atmosphere, J. W. Strohbehn, ed. (Springer-Verlag, New York, 1978).

10. A. Ishimaru, Wave Propagation and Scattering in Random Media, (Academic, New York, 1978), Vol. 2.

11. H. T. Yura, "Short-term average optical-beam spread in a turbulent médium," J. Opt. Soc. Am. 63, 567-572 (1973).

12. H. T. Yura, "Mutual coherence function of a finite cross section optical beam propagating in a turbulent medium," Appl. Opt. 11, 1399-1406 (1972).

13. A. Papoulis, Probability, Random Variables and Stochastic Processes, 2nd ed. (McGraw-Hill, New York, 1984).

14. D. L. Fried, "Statistics of laser beam fade induced by pointing jitter," Appl. Opt. 12, 422-423 (1973).

15. J. N. Pierce, "Theoretical diversity improvement in frequencyshift keying," Proc. IRE 903-910 (1958).

16. Z. Sodnik and R. H. Czichy, "Design data summary of the ESA optical ground station for in-orbit check-out of laser communication payloads and for the observation and registration of space debris," document AD1, ESA ITT AO/1-3930/01/NL/ CK, in ARTEMIS Laser Link for Atmospheric Turbulence Statistics (European Space Agency, 2001).

17. "SP-8-1-1 ARTEMIS satellite performance specification," Sigue 9, Appendix D, (European Space Agency, 1998).

18. A. Comeron, J. A. Rubio, and A. Belmonte, "ASTC inter-island measurement campaign. Final report. Data analysis. Atmospheric modelling. Fade and bit-error-rate statistics" (European Space Agency, July 1996).

19. T. Prud'homme, X. Calbet, E. Garcia, S. Hughes, "Optical ground station atmospheric seeing test campaign. Stellar seeing measurements," Final Report P/GS-RP/96-004 (European Space Agency, April 1996).

20. G. J. Baker and R. S. Benson, "Gaussian beam scintillation on ground to space paths: the importance of beam wander," in Free-Space Laser Communications IV, Proc. SPIE 5550, 225235 (2004).

21. I. S. Gradshteyn, I. M. Ryzhik, and A. Jeffrey, eds., Table of Integrals, Series and Products (Academic, San Diego, 1994). 